\title{
Optimal Dividends under a Ruin Probability Constraint
}

\author{
David C M Dickson and Steve Drekic
}

\begin{abstract}
We consider a classical surplus process modified by the payment of dividends when the insurer's surplus exceeds a threshold. We use a probabilistic argument to obtain general expressions for the expected present value of dividend payments, and show how these expressions can be applied for certain individual claim amount distributions. We then consider the question of maximising the expected present value of dividend payments subject to a constraint on the insurer's ruin probability.
\end{abstract}

Key words: Dividends, ruin probability, time of ruin, severity of ruin. 


\section{Introduction and notation}

In this paper we consider a classical surplus process modified by the payment of dividends from the premium income when the surplus level is above a certain value, which we refer to as the threshold.

We therefore start by introducing some results and notation for the classical surplus process. We denote the classical surplus process by $\{U(t)\}_{t \geq 0}$ where

$$
U(t)=u+c t-\sum_{i=1}^{N(t)} X_{i},
$$

$u \geq 0$ is the initial surplus, $c$ is the rate of premium income per unit time (assumed to be received continuously), $\{N(t)\}_{t \geq 0}$ is a Poisson process with parameter $\lambda$, and $\left\{X_{i}\right\}_{i=1}^{\infty}$ is a sequence of independent and identically distributed positive random variables. For convenience, we assume that $X_{1}$ is a continuous random variable with distribution function $F$, density function $f$, and mean $\mu<\infty$. Let $\theta>0$ be the premium loading factor, so that $c=(1+\theta) \lambda \mu$.

For this surplus process, we define $T_{u}$ to be the time of ruin, so that

$$
T_{u}=\inf \{t \mid U(t)<0\}
$$

with $T_{u}=\infty$ if $U(t) \geq 0$ for all $t>0$. The ultimate ruin probability is given by $\psi(u)=\operatorname{Pr}\left(T_{u}<\infty\right)$.

Central to our subsequent analysis is a special case of the so-called GerberShiu function (see Gerber and Shiu (1998)). We define

$$
\phi(u)=E\left[\exp \left\{-\delta T_{u}-s\left|U\left(T_{u}\right)\right|\right\} I\left(T_{u}<\infty\right)\right]
$$

to be the bivariate Laplace transform of the time of ruin and the severity of ruin, $\left|U\left(T_{u}\right)\right|$. Thus

$$
\phi(u)=\int_{0}^{\infty} \int_{0}^{\infty} e^{-\delta t-s y} w(u, y, t) d y d t
$$

where $w(u, y, t)$ is the defective density of the time $(t)$ and severity $(y)$ of ruin, from initial surplus $u$. We define $w(u, t)$ to be the defective density of the time of ruin, with

$$
w(u, t)=\int_{0}^{\infty} w(u, y, t) d y .
$$

Another key equation in our analysis is Lundberg's fundamental equation, which is

$$
\lambda+\delta-c t=\lambda \int_{0}^{\infty} e^{-t x} f(x) d x
$$


This equation has a unique positive solution for $t$ (see Gerber and Shiu (1998)), and for the claim amount distributions considered in the next section there is at least one negative solution. In this equation we can think of $\delta$ as the Laplace transform parameter in $\phi$, but we can also think of $\delta$ as a force of interest, and we will use this interpretation in the next section.

We now introduce the dividends modification. We assume that when the surplus process is above level $b$, the insurer pays dividends to shareholders at rate $\hat{c}$. Specifically, we assume in Section 2 that $c-\hat{c}>\lambda \mu$, as this condition guarantees that ruin for our modified surplus process does not occur with probability one. Gerber and Shiu (2005) have considered this modified surplus process and, by solving differential equations, have derived expressions for the expected present value of dividend income to shareholders when claims have an exponential or a mixed exponential distribution. Lin and Pavlova (2005) also consider this model and define and study the Gerber-Shiu function. In the next section we will provide an alternative method, based on probabilistic arguments, of finding explicit solutions for the expected present value of dividend payments to shareholders. We illustrate ideas using exponential and mixed exponential claim amount distributions, but the technique can be applied to other distributions as well.

In much of the literature on dividend problems, the focus is on maximising the expected present value of dividend payments to shareholders. See, for example, Gerber and Shiu (2004), Dickson and Waters (2004) and references therein. In Section 3, we retain this focus, but impose a constraint. In particular, we consider maximising the expected present value of dividend payments to shareholders subject to a prescribed level of ruin probability. We present some numerical illustrations and make comparisons with situations in which there is no such constraint. The idea of a constraint is not new. Paulsen (2003) considered a finite time ruin probability constraint while using a diffusion process to model the insurer's surplus. His constraint applied from the time at which dividends first became payable, whereas we shall apply our constraint at time 0 .

\section{Dividends}

In what follows we assume that dividends are payable at rate $\hat{c}$ when the surplus level is above $b$, with $c-\hat{c}>\lambda \mu$, and that if ruin occurs, no further dividends are payable. We will adapt the notation of the previous section by introducing a hat to indicate that the insurer's net rate of premium income is $c-\hat{c}$, rather than $c$. Thus, for example, $\hat{T}_{u}$ denotes the time of ruin for a classical surplus process with initial surplus $u$ and premium rate $c-\hat{c}$. 
Let $V(u, b)$ denote the expected present value of dividend payments at force of interest $\delta$. For $0 \leq u \leq b$, dividends will be payable only if the surplus process reaches $b$ without ruin first occurring, so that

$$
V(u, b)=E\left[\exp \left\{-\delta T_{u, b}\right\}\right] V(b, b)
$$

where $T_{u, b}$ is the time of the first upcrossing of the surplus process through $b$ from $u$ without ruin occurring. See Gerber (1979, p.147).

For $u \geq b$, dividends are payable immediately at rate $\hat{c}$ until the first time the surplus falls below $b$ (an event which may not occur). As this time is identical in distribution to $\hat{T}_{u-b}$, we can write

$$
\begin{aligned}
V(u, b)= & \hat{c} E\left[\bar{a}_{\hat{T}_{u-b}}\right]+\int_{0}^{\infty} e^{-\delta t} \int_{0}^{b} \hat{w}(u-b, y, t) V(b-y, b) d y d t \\
= & \frac{\hat{c}}{\delta}\left(1-E\left[e^{-\delta \hat{T}_{u-b}}\right]\right)+\int_{0}^{\infty} e^{-\delta t} \int_{0}^{b} \hat{w}(u-b, y, t) V(b-y, b) d y d t \\
= & \frac{\hat{c}}{\delta}\left(1-E\left[e^{-\delta \hat{T}_{u-b}}\right]\right) \\
& +V(b, b) \int_{0}^{\infty} e^{-\delta t} \int_{0}^{b} \hat{w}(u-b, y, t) E\left[\exp \left\{-\delta T_{b-y, b}\right\}\right] d y d t
\end{aligned}
$$

In particular,

$$
\begin{aligned}
V(b, b)= & \frac{\hat{c}}{\delta}\left(1-E\left[e^{-\delta \hat{T}_{0}}\right]\right) \\
& +V(b, b) \int_{0}^{\infty} e^{-\delta t} \int_{0}^{b} \hat{w}(0, y, t) E\left[\exp \left\{-\delta T_{b-y, b}\right\}\right] d y d t
\end{aligned}
$$

which gives

$$
V(b, b)=\frac{(\hat{c} / \delta)\left(1-E\left[e^{-\delta \hat{T}_{0}}\right]\right)}{1-\int_{0}^{\infty} e^{-\delta t} \int_{0}^{b} \hat{w}(0, y, t) E\left[\exp \left\{-\delta T_{b-y, b}\right\}\right] d y d t} .
$$

Hence, for $0 \leq u \leq b$,

$$
V(u, b)=\frac{(\hat{c} / \delta)\left(1-E\left[e^{-\delta \hat{T}_{0}}\right]\right) E\left[\exp \left\{-\delta T_{u, b}\right\}\right]}{1-\int_{0}^{\infty} e^{-\delta t} \int_{0}^{b} \hat{w}(0, y, t) E\left[\exp \left\{-\delta T_{b-y, b}\right\}\right] d y d t}
$$

At first sight, it appears that in order to apply formulae (2) and (3) we need to know the functional form of $\hat{w}(u, y, t)$. However, as we shall see in the subsequent analysis, this need not be the case. 


\subsection{Exponential claims}

When the claim amount distribution is exponential, which we denote by $f(x)=\alpha e^{-\alpha x}$, it is relatively straightforward to obtain expressions for $V(u, b)$. In large part, this is due to the fact that $\hat{w}(u, y, t)=\hat{w}(u, t) f(y)$ due to the memoryless property of the exponential distribution.

For this claim amount distribution, we know from Gerber (1979, p.147) that

$$
E\left[\exp \left\{-\delta T_{u, b}\right\}\right]=\frac{m(u)}{m(b)}
$$

where

$$
m(u)=(\alpha+\rho) e^{\rho u}-(\alpha-R) e^{-R u}
$$

and $\rho>0$ and $-R<0$ (which depend on $\delta$ ) are the solutions of Lundberg's fundamental equation (1), i.e.

$$
\lambda+\delta-c t=\frac{\lambda \alpha}{\alpha+t} .
$$

Hence, for $0 \leq u \leq b$, we get

$$
V(u, b)=\frac{(\hat{c} / \delta)\left(1-E\left[e^{-\delta \hat{T}_{0}}\right]\right) m(u)}{m(b)-\int_{0}^{\infty} e^{-\delta t} \int_{0}^{b} \hat{w}(0, y, t) m(b-y) d y d t} .
$$

Considering first the numerator, and noting that for $u \geq 0$

$$
E\left[\exp \left\{-\delta T_{u}\right\} I\left(T_{u}<\infty\right)\right]=E\left[\exp \left\{-\delta T_{u}\right\}\right]
$$

(see Gerber and Shiu (1998)), we have

$$
E\left[e^{-\delta \hat{T}_{0}}\right]=\int_{0}^{\infty} e^{-\delta t} \hat{w}(0, t) d t=1-\hat{R} / \alpha
$$

where the second identity follows from formula (5.38) of Gerber and Shiu (1998), and $-\hat{R}<0$ is the negative solution of Lundberg's fundamental equation with premium rate $c-\hat{c}$. Next, let

$$
\begin{aligned}
L(b) & =m(b)-\int_{0}^{\infty} e^{-\delta t} \int_{0}^{b} \hat{w}(0, y, t) m(b-y) d y d t \\
& =m(b)-\int_{0}^{\infty} e^{-\delta t} \hat{w}(0, t) \int_{0}^{b} \alpha e^{-\alpha y} m(b-y) d y d t \\
& =m(b)-\left(\int_{0}^{\infty} e^{-\delta t} \hat{w}(0, t) d t\right) \alpha e^{-\alpha b} \int_{0}^{b} e^{\alpha y} m(y) d y .
\end{aligned}
$$


Then

$$
\begin{aligned}
\int_{0}^{b} e^{\alpha y} m(y) d y & =\int_{0}^{b} e^{\alpha y}\left((\alpha+\rho) e^{\rho y}-(\alpha-R) e^{-R y}\right) d y \\
& =e^{(\alpha+\rho) b}-e^{(\alpha-R) b}
\end{aligned}
$$

so that

$$
\begin{aligned}
L(b) & =(\alpha+\rho) e^{\rho b}-(\alpha-R) e^{-R b}-(\alpha-\hat{R})\left(e^{\rho b}-e^{-R b}\right) \\
& =(\rho+\hat{R}) e^{\rho b}+(R-\hat{R}) e^{-R b}
\end{aligned}
$$

and hence

$$
V(u, b)=\frac{\hat{c} \hat{R}}{\delta \alpha} \frac{(\alpha+\rho) e^{\rho u}-(\alpha-R) e^{-R u}}{(\rho+\hat{R}) e^{\rho b}+(R-\hat{R}) e^{-R b}} .
$$

Consider now the case when $u \geq b$. Writing $\varphi(u-b)=E\left[e^{-\delta \hat{T}_{u-b}}\right]$, the first term in formula (2) involves

$$
\varphi(u-b)=\int_{0}^{\infty} e^{-\delta t} \hat{w}(u-b, t) d t=\varphi(0) e^{-\hat{R}(u-b)} .
$$

See, for example, Gerber and Shiu (1998, equation (3.16)). Then for $u \geq b$,

$$
\begin{aligned}
V(u, b)= & \frac{\hat{c}}{\delta}\left(1-E\left[e^{-\delta \hat{T}_{u-b}}\right]\right) \\
& +\int_{0}^{\infty} e^{-\delta t} \hat{w}(u-b, t) d t \int_{0}^{b} f(y) \frac{m(b-y)}{m(b)} V(b, b) d y \\
= & \frac{\hat{c}}{\delta}(1-\varphi(u-b))+\varphi(u-b) \frac{V(b, b)}{m(b)} \alpha\left(e^{\rho b}-e^{-R b}\right) .
\end{aligned}
$$

In particular,

$$
V(b, b)=\frac{\hat{c}}{\delta}(1-\varphi(0))+\varphi(0) \frac{V(b, b)}{m(b)} \alpha\left(e^{\rho b}-e^{-R b}\right)
$$

gives

$$
\frac{V(b, b)}{m(b)} \alpha\left(e^{\rho b}-e^{-R b}\right)=\frac{1}{\varphi(0)}\left(V(b, b)-\frac{\hat{c}}{\delta}(1-\varphi(0))\right)
$$

so that

$$
\begin{aligned}
V(u, b) & =\frac{\hat{c}}{\delta}(1-\varphi(u-b))+\frac{\varphi(u-b)}{\varphi(0)}\left(V(b, b)-\frac{\hat{c}}{\delta}(1-\varphi(0))\right) \\
& =\frac{\hat{c}}{\delta}\left(1-e^{-\hat{R}(u-b)}\right)+e^{-\hat{R}(u-b)} V(b, b) .
\end{aligned}
$$


For $0 \leq u \leq b$, the value of $b$ that maximises $V(u, b)$ is the value of $b$ that minimises $L(b)$. Thus

$$
\frac{\partial}{\partial b} L(b)=(\rho+\hat{R}) \rho e^{\rho b}-(R-\hat{R}) R e^{-R b}
$$

and setting this equal to 0 gives the optimal level, $b^{*}$, as

$$
b^{*}=\frac{1}{\rho+R} \log \frac{(R-\hat{R}) R}{(\rho+\hat{R}) \rho} .
$$

We remark that the formulae for $V(u, b)$ and $b^{*}$ can be found in Gerber and Shiu (2005).

Although the exponential distribution is the most straightforward claim amount distribution to consider, one advantage it offers is that it is the basis of De Vylder's (1978) approximation, and Højgaard (2002) has shown that this approximation can successfully be applied to an optimal dividends problem. As the solution to the problem considered in Section 3 is considerably easier to deal with for exponential claims than for other claim amount distributions, De Vylder's approximation has a certain appeal.

\subsection{Mixed exponential claims}

Let us now consider the case when the claim amount distribution is a mixture of two exponentials with

$$
f(x)=p \alpha e^{-\alpha x}+q \beta e^{-\beta x}
$$

where $p>0$ and $p+q=1$.

Following the arguments in Gerber (1979, pp.147-148) we find that, as in the previous case,

$$
E\left[\exp \left\{-\delta T_{u, b}\right\}\right]=\frac{m(u)}{m(b)}
$$

where we now have

$$
\frac{m(u)}{m(b)}=\varpi_{0}(\delta, b) e^{\rho u}+\varpi_{1}(\delta, b) e^{-R_{1} u}+\varpi_{2}(\delta, b) e^{-R_{2} u}
$$

where $\rho>0,-R_{1}<0$ and $-R_{2}<0$ (which all depend on $\delta$ ) are the solutions of Lundberg's fundamental equation (1), i.e.

$$
\lambda+\delta-c t=\frac{\lambda p \alpha}{\alpha+t}+\frac{\lambda q \beta}{\beta+t} .
$$


The coefficients $\left\{\varpi_{i}(\delta, b)\right\}_{i=0}^{2}$ satisfy the conditions

$$
\begin{aligned}
1 & =\varpi_{0}(\delta, b) e^{\rho b}+\varpi_{1}(\delta, b) e^{-R_{1} b}+\varpi_{2}(\delta, b) e^{-R_{2} b}, \\
0 & =\frac{\varpi_{0}(\delta, b)}{\alpha+\rho}+\frac{\varpi_{1}(\delta, b)}{\alpha-R_{1}}+\frac{\varpi_{2}(\delta, b)}{\alpha-R_{2}}, \\
0 & =\frac{\varpi_{0}(\delta, b)}{\beta+\rho}+\frac{\varpi_{1}(\delta, b)}{\beta-R_{1}}+\frac{\varpi_{2}(\delta, b)}{\beta-R_{2}},
\end{aligned}
$$

where the final two conditions are just a special case of equation (A9) of Gerber and Shiu (2005).

Considering first the case when $0 \leq u \leq b$, in the numerator of formula (3) for $V(u, b)$ we have

$$
E\left[e^{-\delta \hat{T}_{0}}\right]=\frac{\lambda}{c-\hat{c}}\left(\frac{p}{\alpha+\hat{\rho}}+\frac{q}{\beta+\hat{\rho}}\right)
$$

(see Dickson (2005, p.188)) where $\hat{\rho}>0$ is the positive solution of Lundberg's fundamental equation with premium rate $c-\hat{c}$. To find the denominator of formula (3) for $V(u, b)$ we shall find the double integral by treating it as a special case of the double integral in formula (2). The starting point is the function $\phi$, defined in Section 1. From Gerber and Shiu (1998), this function satisfies the equation

$$
c \phi^{\prime}(u)=(\delta+\lambda) \phi(u)-\lambda \int_{0}^{u} \phi(x) f(u-x) d x-\lambda \omega(u)
$$

where

$$
\begin{aligned}
\omega(u) & =\int_{0}^{\infty} e^{-s y} f(u+y) d y \\
& =\frac{p \alpha}{\alpha+s} e^{-\alpha u}+\frac{q \beta}{\beta+s} e^{-\beta u} .
\end{aligned}
$$

By applying standard arguments (see Gerber $(1979$, p.117)) we find that

$$
\phi(u)=k_{0}(\delta, s) e^{\rho u}+k_{1}(\delta, s) e^{-R_{1} u}+k_{2}(\delta, s) e^{-R_{2} u} .
$$

As $\rho>0$ and $\lim _{u \rightarrow \infty} \phi(u)=0$, we must have that $k_{0}(\delta, s)=0$. Next, we can obtain $k_{1}(\delta, s)$ and $k_{2}(\delta, s)$ by inserting expression (8) for $\phi$ into equation (7). We obtain the equalities

$$
\begin{aligned}
& \frac{1}{\alpha+s}=\frac{k_{1}(\delta, s)}{\alpha-R_{1}}+\frac{k_{2}(\delta, s)}{\alpha-R_{2}} \\
& \frac{1}{\beta+s}=\frac{k_{1}(\delta, s)}{\beta-R_{1}}+\frac{k_{2}(\delta, s)}{\beta-R_{2}}
\end{aligned}
$$


which give

$$
\begin{aligned}
& k_{1}(\delta, s)=\gamma_{1}(\delta) \frac{\alpha}{\alpha+s}+\gamma_{2}(\delta) \frac{\beta}{\beta+s}, \\
& k_{2}(\delta, s)=\sigma_{1}(\delta) \frac{\alpha}{\alpha+s}+\sigma_{2}(\delta) \frac{\beta}{\beta+s},
\end{aligned}
$$

where

$$
\begin{aligned}
\gamma_{1}(\delta) & =\frac{\left(\alpha-R_{1}\right)\left(\alpha-R_{2}\right)\left(\beta-R_{1}\right)}{\alpha\left(R_{2}-R_{1}\right)(\alpha-\beta)}, \\
\gamma_{2}(\delta) & =-\frac{\left(\alpha-R_{1}\right)\left(\beta-R_{1}\right)\left(\beta-R_{2}\right)}{\beta\left(R_{2}-R_{1}\right)(\alpha-\beta)} \\
\sigma_{1}(\delta) & =-\frac{\left(\alpha-R_{1}\right)\left(\alpha-R_{2}\right)\left(\beta-R_{2}\right)}{\alpha\left(R_{2}-R_{1}\right)(\alpha-\beta)} \\
\sigma_{2}(\delta) & =\frac{\left(\alpha-R_{2}\right)\left(\beta-R_{1}\right)\left(\beta-R_{2}\right)}{\beta\left(R_{2}-R_{1}\right)(\alpha-\beta)}
\end{aligned}
$$

Thus

$$
\begin{aligned}
\phi(u)= & \left(\gamma_{1}(\delta) e^{-R_{1} u}+\sigma_{1}(\delta) e^{-R_{2} u}\right) \frac{\alpha}{\alpha+s} \\
& +\left(\gamma_{2}(\delta) e^{-R_{1} u}+\sigma_{2}(\delta) e^{-R_{2} u}\right) \frac{\beta}{\beta+s},
\end{aligned}
$$

so that for $u \geq 0$,

$$
w(u, y, t)=\eta_{1}(u, t) \alpha e^{-\alpha y}+\eta_{2}(u, t) \beta e^{-\beta y}
$$

where for $i=1,2$,

$$
\int_{0}^{\infty} e^{-\delta t} \eta_{i}(u, t) d t=\gamma_{i}(\delta) e^{-R_{1} u}+\sigma_{i}(\delta) e^{-R_{2} u}
$$

In the context of finding the expected present value of dividend payments, we need to evaluate

$$
\int_{0}^{\infty} e^{-\delta t} \int_{0}^{b} \hat{w}(u-b, y, t) m(b-y) d y d t
$$


for $u \geq b$. This can be written as

$$
\begin{aligned}
& \int_{0}^{\infty} e^{-\delta t} \int_{0}^{b}\left(\hat{\eta}_{1}(u-b, t) \alpha e^{-\alpha y}+\hat{\eta}_{2}(u-b, t) \beta e^{-\beta y}\right) m(b-y) d y d t \\
= & \int_{0}^{\infty} e^{-\delta t} \hat{\eta}_{1}(u-b, t) d t \int_{0}^{b} \alpha e^{-\alpha y} m(b-y) d y \\
& +\int_{0}^{\infty} e^{-\delta t} \hat{\eta}_{2}(u-b, t) d t \int_{0}^{b} \beta e^{-\beta y} m(b-y) d y \\
= & \left(\hat{\gamma}_{1}(\delta) e^{-\hat{R}_{1}(u-b)}+\hat{\sigma}_{1}(\delta) e^{-\hat{R}_{2}(u-b)}\right) \int_{0}^{b} \alpha e^{-\alpha y} m(b-y) d y \\
& +\left(\hat{\gamma}_{2}(\delta) e^{-\hat{R}_{1}(u-b)}+\hat{\sigma}_{2}(\delta) e^{-\hat{R}_{2}(u-b)}\right) \int_{0}^{b} \beta e^{-\beta y} m(b-y) d y .
\end{aligned}
$$

The integrals in the above expression are straightforward to evaluate as $m$ is a linear combination of three exponential functions.

Then for $0 \leq u \leq b$, we have all the components required to find $V(u, b)$. For $u \geq b$, we require an additional component, namely $E\left[e^{-\delta \hat{T}_{u-b}}\right]$. This is easily obtained from the above workings by noting that

$$
\left.\phi(u)\right|_{s=0}=E\left[\exp \left\{-\delta T_{u}\right\} I\left(T_{u}<\infty\right)\right]
$$

so that by formulae (4) and (9), we have

$$
E\left[\exp \left\{-\delta T_{u}\right\}\right]=\left(\gamma_{1}(\delta)+\gamma_{2}(\delta)\right) e^{-R_{1} u}+\left(\sigma_{1}(\delta)+\sigma_{2}(\delta)\right) e^{-R_{2} u}
$$

We remark that, unlike in the case of exponential claims, it does not seem possible to obtain a closed form solution for the level $b^{*}$ that maximises the expected present value of dividend payments for given values of $u$ and $\hat{c}$. However, it is possible to find this level numerically.

\subsection{An alternative approach}

In the previous subsection, we derived expressions for $V(u, b)$ both for $0 \leq u \leq b$ and $u \geq b$. If we are interested only in the former case, there is an alternative, more straightforward approach to finding the double integral in formula (3).

From Gerber and Shiu (1998, equation (2.6)) we know that

$$
\phi(0)=\frac{\lambda}{c} \int_{0}^{\infty} e^{-\rho t} \int_{t}^{\infty} e^{-s(y-t)} f(y) d y d t
$$


where $\rho>0$ is the unique positive solution of Lundberg's fundamental equation (1), and hence depends on $\delta$. Consider a more general mixed exponential distribution than in the previous subsection, say

$$
f(x)=\sum_{i=1}^{n} w_{i} \alpha_{i} \exp \left\{-\alpha_{i} x\right\}
$$

where $\left\{w_{i}\right\}_{i=1}^{n}$ are positive weights which sum to 1 . Then

$$
\begin{aligned}
\phi(0) & =\frac{\lambda}{c} \sum_{i=1}^{n} w_{i} \alpha_{i} \int_{0}^{\infty} e^{-\rho t} \int_{t}^{\infty} e^{-s(y-t)} e^{-\alpha_{i} y} d y d t \\
& =\frac{\lambda}{c} \sum_{i=1}^{n} w_{i} \alpha_{i} \int_{0}^{\infty} e^{-(\rho-s) t} \frac{e^{-\left(s+\alpha_{i}\right) t}}{s+\alpha_{i}} d t \\
& =\frac{\lambda}{c} \sum_{i=1}^{n} w_{i} \frac{\alpha_{i}}{s+\alpha_{i}} \frac{1}{\rho+\alpha_{i}} .
\end{aligned}
$$

Thus, we have

$$
w(0, y, t)=\sum_{i=1}^{n} \eta_{i}(t) \alpha_{i} \exp \left\{-\alpha_{i} y\right\}
$$

where for $i=1,2, \ldots, n$,

$$
\int_{0}^{\infty} e^{-\delta t} \eta_{i}(t) d t=\frac{\lambda}{c} \frac{w_{i}}{\rho+\alpha_{i}}
$$

In the case when $n=2$, we thus obtain slightly simpler coefficients than in the previous subsection, as we have

$$
\gamma_{1}(\delta)+\sigma_{1}(\delta)=\frac{\left(\alpha-R_{1}\right)\left(\alpha-R_{2}\right)}{\alpha(\alpha-\beta)}=\frac{\lambda}{c} \frac{p}{\alpha+\rho}
$$

and

$$
\gamma_{2}(\delta)+\sigma_{2}(\delta)=-\frac{\left(\beta-R_{1}\right)\left(\beta-R_{2}\right)}{\beta(\alpha-\beta)}=\frac{\lambda}{c} \frac{q}{\beta+\rho} .
$$

We remark that these last two equalities also lead to formula (6) (allowing for the different premium rate).

\section{Dividends under a constraint}

In this section we turn our attention to the expected present value of dividend payments subject to a ruin probability constraint. We motivate the problem 
in the following way. Suppose that the insurer is not paying dividends and has an initial surplus which gives a ruin probability below a regulated level (e.g. the regulated level is $1 \%$, and the insurer's initial surplus, $u$, is such that $\psi(u)=0.005)$. Then the insurer is in a position to pay dividends to shareholders and still satisfy the regulations on ruin probability. For example, for $u$ such that $\psi(u)=0.005$, there is a set of pairs of dividend rate and threshold that result in a ruin probability of 0.01. Our objective is then to find the optimal pair, where optimal means that the expected present value of dividend payments is maximised, subject to the constraint on the ruin probability. We will use the notation $\psi(u, b)$ to denote the ultimate ruin probability when the dividend threshold is $b$.

Our aim here is not to derive explicit solutions for optimal levels. Indeed, it is not clear that this is possible, even in the simplest situation (exponential claims). Rather we wish to investigate the effect of the constraint, and so all results presented in this section have been obtained numerically, using the software Mathematica. Ruin probabilities when dividends are payable have been calculated using results given in Dickson (1991).

As a first illustration, let us consider the case when the claim amount distribution is exponential with mean 1 . We set $\lambda=1$ and $\delta=0.001$, so that we can think of this as a force of interest of $10 \%$ per annum with 100 claims expected per annum. Now suppose that the premium is calculated with a $20 \%$ loading, and that $u=30.7$ so that $\psi(u)=0.005$ (using the well-known formula for $\psi$ in this case). Let us further suppose that the insurer is subject to a regulated ruin probability of $\varepsilon=0.01$.

In this situation, we find that the optimal combination of $b$ and $\hat{c}$ such that $\psi(u, b)=0.01$ is $b^{\#}=49.1$ and $\hat{c}^{\#}=0.1912$, giving $V\left(u, b^{\#}\right)=153.76$. Let us compare this with two other scenarios, under each of which the insurer is not subject to a ruin probability constraint. First, suppose that the insurer selects the optimal level of dividends, $\hat{c}^{\#}=0.1912$. Then, by formula (5), the optimal threshold is $b^{*}=27.11$, giving $V\left(u, b^{*}\right)=166.46$ and $\psi\left(u, b^{*}\right)=$ 0.167 . Second, suppose that the insurer simply elects to maximise $V(u, b)$ subject to the constraint $c-\hat{c} \geq \lambda \mu$. Then the optimal threshold is $\bar{b}=27.96$, the optimal dividend rate is $\bar{c}=0.2, V(u, \bar{b})=170.5$, and $\psi(u, \bar{b})=1$.

Looking at these numbers, we can see that in the first case, for the same rate of dividend payment, the relaxation of the ruin probability constraint has produced about an $8 \%$ increase in the expected present value of dividend payments, but the ruin probability has increased by a factor of almost 17 . The second change results in about an $11 \%$ increase in the expected present value of dividend payments, while the ruin probability has increased by a factor of 100 .

Table 1 shows results for a range of scenarios, labelled (A) to (G). In 


\begin{tabular}{ccccccccc} 
Scenario & $c$ & $\delta$ & $u$ & $\psi(u)$ & $\varepsilon$ & $V\left(u, b^{\#}\right)$ & $b^{\#}$ & $\hat{c}^{\#}$ \\
\hline \hline (A) & 1.1 & 0.001 & 57.23 & 0.005 & 0.01 & 58.30 & 77.66 & 0.0866 \\
(B) & 1.2 & 0.001 & 30.70 & 0.005 & 0.01 & 153.76 & 49.10 & 0.1912 \\
(C) & 1.3 & 0.001 & 21.82 & 0.005 & 0.01 & 253.23 & 38.14 & 0.2933 \\
(D) & 1.1 & 0.001 & 49.61 & 0.01 & 0.025 & 60.64 & 65.49 & 0.0867 \\
(E) & 1.1 & 0.001 & 49.61 & 0.01 & 0.05 & 66.96 & 54.68 & 0.0870 \\
(F) & 1.1 & 0.002 & 57.23 & 0.005 & 0.01 & 23.88 & 70.38 & 0.0769 \\
(G) & 1.1 & 0.003 & 57.23 & 0.005 & 0.01 & 14.12 & 65.83 & 0.0688 \\
\hline
\end{tabular}

Table 1: Optimal levels and expected present values.

this table, the optimal threshold $\left(b^{\#}\right)$ and dividend rate $\left(\hat{c}^{\#}\right)$ are shown for a given level of ruin probability $(\varepsilon)$, together with the expected present value of dividend payments. In Table 2 we show the optimal threshold $b^{*}$, calculated by formula (5) when the dividend rate is $\hat{c}^{\#}$ (from Table 1), together with the resulting expected present value of dividend payments $\left(V\left(u, b^{*}\right)\right)$ and ruin probability $\left(\psi\left(u, b^{*}\right)\right)$. In Table 3 we show the optimal values of the threshold $(\bar{b})$ and the dividend rate $(\bar{c})$ when the objective is simply to maximise the expected present value of dividend payments subject to the constraint $c-\hat{c} \geq$ $\lambda \mu$. The maximum expected present value is shown $(V(u, \bar{b}))$ along with the ruin probability $(\psi(u, \bar{b}))$.

\begin{tabular}{ccccc} 
Scenario & $u$ & $b^{*}$ & $V\left(u, b^{*}\right)$ & $\psi\left(u, b^{*}\right)$ \\
\hline \hline (A) & 57.23 & 24.34 & 79.12 & 0.293 \\
(B) & 30.70 & 27.11 & 166.46 & 0.167 \\
(C) & 21.82 & 25.02 & 260.90 & 0.099 \\
(D) & 49.61 & 24.36 & 76.65 & 0.325 \\
(E) & 49.61 & 24.41 & 76.82 & 0.330 \\
(F) & 57.23 & 11.84 & 37.04 & 0.237 \\
(G) & 57.23 & 5.55 & 22.54 & 0.167 \\
\hline
\end{tabular}

Table 2: Levels assuming dividend payments at rate $\hat{c}^{\#}$.

Considering first Table 1, we see that the optimal dividend rate is fairly close to the premium loading factor $\theta$ when $\delta=0.001$, but not for the other values of $\delta$. Indeed, both $\hat{c}^{\#}$ and $b^{\#}$ decrease as $\delta$ increases. We also note that in each scenario, the optimal threshold is above $u$.

In Table 2, we observe that the ruin probabilities are much higher than the corresponding $\varepsilon$ values in Table 1, and an increase in either the premium rate (scenarios $(\mathrm{A})$ to $(\mathrm{C})$ ) or the force of interest (scenarios $(\mathrm{A}),(\mathrm{F})$ and $(\mathrm{G})$ ) results in a reduced ruin probability. As formula (5) for $b^{*}$ is independent 


\begin{tabular}{cccccc} 
Scenario & $u$ & $\bar{b}$ & $\bar{c}$ & $V(u, \bar{b})$ & $\psi(u, \bar{b})$ \\
\hline \hline (A) & 57.23 & 26.82 & 0.1 & 87.54 & 1 \\
(B) & 30.70 & 27.96 & 0.2 & 170.50 & 1 \\
(C) & 21.82 & 25.48 & 0.3 & 263.99 & 1 \\
(D) & 49.61 & 26.82 & 0.1 & 84.20 & 1 \\
(E) & 49.61 & 26.82 & 0.1 & 84.20 & 1 \\
(F) & 57.23 & 15.01 & 0.1 & 46.39 & 1 \\
(G) & 57.23 & 9.24 & 0.1 & 31.88 & 1 \\
\hline
\end{tabular}

Table 3: Levels under the constraint $c-\hat{c} \geq \lambda \mu$.

of $u$, there is no relationship between the threshold and initial surplus as there is in Table 1. In each scenario the expected present value of dividend payments is greater than in Table 1 , since $b^{*}$ is the optimal threshold.

In Table 3 we see that the optimal dividend level is always equal to the premium loading factor, which results in certain ruin. The expected present value of dividend payments is greater under each scenario than in Table 2, and hence under Table 1.

Thus, the changes as we move from Table 1 to Table 3 result in both increased ruin probabilities and increased expected present values of dividend payments. Scenario (C) is perhaps the most interesting. Here we see that the expected present values in Tables 2 and 3 are respectively $3 \%$ and $4.2 \%$ greater than in Table 1, while the ruin probabilities have increased by factors of 9.9 and 100. It is interesting that relaxing the ruin probability constraint benefits the shareholders so little in this scenario.

As a second illustration, we consider the situation when the claim amount distribution is a mixed exponential distribution with $f(x)=\frac{2}{3}\left(2 e^{-2 x}\right)+$ $\frac{1}{3}\left(\frac{1}{2} e^{-x / 2}\right)$ so that $\mu=1$. Table 4 shows values for the same scenarios as in Table 1, and we observe a similar pattern as in Table 1. However, in this case the calculations are not as straightforward as those for Table 1. In particular, the solutions of Lundberg's fundamental equation do not exist in a neat closed form as they do when the claim amount distribution is exponential. Table 5 shows the values we obtain when we use De Vylder's approximation to the surplus process, resulting from the same simple calculations that produce Table 1. These approximations are remarkably good, suggesting that in cases where explicit solutions for $V(u, b)$ exist, at the very least the use of De Vylder's approximation provides an excellent indication of the levels of the optimal pair $\left(b^{\#}, \hat{c}^{\#}\right)$ and $V\left(u, b^{\#}\right)$. 


\begin{tabular}{ccccccccc} 
Scenario & $c$ & $\delta$ & $u$ & $\psi(u)$ & $\varepsilon$ & $V\left(u, b^{\#}\right)$ & $b^{\#}$ & $\hat{c}^{\#}$ \\
\hline \hline (A) & 1.1 & 0.001 & 87.29 & 0.005 & 0.01 & 51.87 & 111.77 & 0.0812 \\
(B) & 1.2 & 0.001 & 47.49 & 0.005 & 0.01 & 143.28 & 72.31 & 0.1870 \\
(C) & 1.3 & 0.001 & 34.17 & 0.005 & 0.01 & 241.42 & 57.04 & 0.2897 \\
(D) & 1.1 & 0.001 & 75.61 & 0.01 & 0.025 & 55.10 & 93.07 & 0.0813 \\
(E) & 1.1 & 0.001 & 75.61 & 0.01 & 0.05 & 63.90 & 76.34 & 0.0815 \\
(F) & 1.1 & 0.002 & 87.29 & 0.005 & 0.01 & 21.14 & 100.01 & 0.0682 \\
(G) & 1.1 & 0.003 & 87.29 & 0.005 & 0.01 & 12.77 & 92.22 & 0.0575 \\
\hline
\end{tabular}

Table 4: Optimal levels and expected present values, mixed exponential claims.

\begin{tabular}{ccccccccc} 
Scenario & $c$ & $\delta$ & $u$ & $\psi(u)$ & $\varepsilon$ & $V\left(u, b^{\#}\right)$ & $b^{\#}$ & $\hat{c}^{\#}$ \\
\hline \hline (A) & 1.1 & 0.001 & 87.25 & 0.005 & 0.01 & 51.88 & 111.72 & 0.0812 \\
(B) & 1.2 & 0.001 & 47.41 & 0.005 & 0.01 & 143.32 & 72.20 & 0.1870 \\
(C) & 1.3 & 0.001 & 34.07 & 0.005 & 0.01 & 241.52 & 56.85 & 0.2898 \\
(D) & 1.1 & 0.001 & 75.58 & 0.01 & 0.025 & 55.11 & 93.04 & 0.0813 \\
(E) & 1.1 & 0.001 & 75.58 & 0.01 & 0.05 & 63.90 & 76.32 & 0.0815 \\
(F) & 1.1 & 0.002 & 87.25 & 0.005 & 0.01 & 21.14 & 99.98 & 0.0683 \\
(G) & 1.1 & 0.003 & 87.25 & 0.005 & 0.01 & 12.77 & 92.20 & 0.0575 \\
\hline
\end{tabular}

Table 5: De Vylder approximations to values in Table 4.

\section{Concluding remarks}

Although our analysis in Section 2 involved the defective density $w(u, y, t)$ we did not need to find its explicit form, although we did identify its general form for the examples considered. It is possible to identify the explicit form of $w(u, y, t)$ for certain claim amount distributions, particularly when $u=0$, and this will be discussed in a forthcoming manuscript.

\section{References}

[1] De Vylder, F. (1978) A practical solution to the problem of ultimate ruin probability. Scandinavian Actuarial Journal, 114-119.

[2] Dickson, D.C.M. (1991) The probability of ultimate ruin with a variable premium loading - a special case. Scandinavian Actuarial Journal, 75-86.

[3] Dickson, D.C.M. (2005) Insurance Risk and Ruin. Cambridge University Press, Cambridge. 
[4] Dickson, D.C.M. and Waters, H.R. (2004) Some optimal dividends problems. ASTIN Bulletin 34, 49-74.

[5] Gerber, H.U. (1979) An Introduction to Mathematical Risk Theory. S.S. Huebner Foundation, Philadelphia, PA.

[6] Gerber, H.U. and Shiu, E.S.W. (1998) On the time value of ruin. North American Actuarial Journal 2, 1, 48-72.

[7] Gerber, H. U. and Shiu, E. S. W. (2004) Optimal dividends: analysis with Brownian motion. North American Actuarial Journal 8, 1, 1-20.

[8] Gerber, H.U. and Shiu, E.S.W. (2005) On optimal dividend strategies in the compound Poisson model. Unpublished manuscript.

[9] Højgaard, B. (2002) Optimal dynamic premium control in non-life insurance. Maximising dividend pay-outs. Scandinavian Actuarial Journal, 225-245.

[10] Lin, X.S. and Pavlova, K. (2005) The compound Poisson risk model with a threshold dividend strategy. Unpublished manuscript.

[11] Paulsen, J. (2003) Optimal dividend payouts for diffusions with solvency constraints. Finance and Stochastics 7, 457-473.

David C M Dickson

Centre for Actuarial Studies

Department of Economics

University of Melbourne

Victoria 3010

Australia

email: dcmd@unimelb.edu.au

\section{Steve Drekic}

Department of Statistics and

Actuarial Science

University of Waterloo

Waterloo, Ontario

Canada N2L 3G1

email: sdrekic@math.uwaterloo.ca 


\section{University Library}

\section{- M M I E R R V A gateway to Melbourne's research publications}

Minerva Access is the Institutional Repository of The University of Melbourne

Author/s:

Dickson, David CM;Drekic, Steve

Title:

Optimal Dividends under a Ruin ProbabilityConstraint

Date:

2005-10

Citation:

Dickson, David CM and Drekic, Steve (2005) Optimal Dividends under a Ruin ProbabilityConstraint.

Persistent Link:

http://hdl.handle.net/11343/34327 